\title{
Anesthesia for Tonsillectomy in a Child with Klippel-Feil Syndrome Associated with Down Syndrome. Case Report
}

\author{
Magda Lourenço Fernandes, TSA ${ }^{1}$, Núbia Campos Faria ${ }^{2}$, Thiago Ferreira Gonçalves ${ }^{3}$, \\ Bruno Holanda Santos 4
}

\begin{abstract}
Summary: Fernandes ML, Faria NC, Gonçalves TF, Santos BH - Anesthesia for Tonsillectomy in a Child with Klippel-Feil Syndrome Associated with Down Syndrome. Case Report.

Background and objectives: Craniofacial abnormalities present in Klippel-Feil Syndrome (KFS) and Down Syndrome (DS) can hinder access to the airways. Oropharyngeal surgeries also require special attention with the airways. The association of both syndromes in a patient scheduled for tonsillectomy is a rare condition that imposes challenges to the anesthetic-surgical treatment. The objective of this report was to discuss the approach of the airways and the risks of cervical manipulation in a patient with KFS and DS undergoing tonsillectomy.
\end{abstract}

Case report: This is a 5 years old child with diagnosis of KFS and DS and instability of the atlantoaxial joint who underwent tonsillectomy under general balanced anesthesia. Ventilation under face mask and tracheal intubation were done with the neck in the neutral position. The perfect visualization of the epiglottis and vocal cords allowed tracheal intubation with conventional laryngoscopy. The surgery was also performed without cervical extension and without intercurrences.

Conclusions: Although access to the airways can be easy, anatomical changes presuppose the presence of difficult airways in patients with KFS and DS. Differentiated care and adequate resources are mandatory to avoid complications during approach of the airways. Cervical manipulation should be avoided in the presence of instability of the atlantoaxial joint due to the risk of neurological damage.

Keywords: DISEASES, Genetic: Klippel-Feil syndrome, Down syndrome; SURGERY, Otorhinolaryngological; TRACHEAL INTUBATION.

[Rev Bras Anestesiol 2010;60(3): 315-320] @Elsevier Editora Ltda

\section{INTRODUCTION}

Craniofacial abnormalities hinder access to the airways, representing a challenge for the anesthesiologist. Klippel-Feil Syndrome (KFS) and Down Syndrome (DS) are examples of this clinical situation. Oropharyngeal interventions also compromise access to the airways. When approaching the airways, all the structures involved should be considered, including the oral cavity, the anterior mandibular space, maxilla, temporomandibular joint, and spine ${ }^{1}$. The objective of this report was to discuss the particularities of the approach of the airways, focusing on anatomical changes and risks of cervical

Received from the CET/SBA of the Santa Casa de Belo Horizonte, MG

1. Anesthesiologist of the Santa Casa de Belo Horizonte and of the Hospital das Clínicas da UFMG; Responsible for the CET/SBA of the Santa Casa de Belo Horizonte; Scientific Director of the SAMG; Member of the Committee of Pediatric Anesthesiology of the SBA; Specialist in Intensive Care

2. Anesthesiologist of the Santa Casa de Belo Horizonte

3. ME3 of the CET/SBA of the Santa Casa de Belo Horizonte

4. Otorhinolaryngologist of the Santa Casa de Belo Horizonte

Submitted on August 23, 2009

Approved on February 8, 2010

Correspondence to:

Dra. Magda Lourenço Fernandes

Rua Padre Marinho, 480/1206

Santa Efigênia

30140-040 - Belo Horizonte, MG, Brasil

E-mail: balourenco@hotmail.com manipulation, in a patient with KFS associated with DS who underwent tonsillectomy.

\section{CASE REPORT}

This is a five years old patient weighing $17 \mathrm{~kg}$, ASA II, with KFS and DS, and the diagnosis of hypertrophy of the palatine tonsils. The patient developed obstructive sleep apnea,confirmed by polysonography indicating the need of tonsillectomy. In the pre-anesthetic evaluation her mother presented a neurology report with the diagnosis of KFS, DS, and instability of the atlantoaxial joint. On physical exam, the patient had a short neck deviated to the left, low hairline at the back of the neck, right scapula elevated and shortened, characterizing Sprengel deformity. She had an oral opening of $2 \mathrm{~cm}$, Mallampati $\mathrm{I}$, and important limitation of cervical extension and rotation. $\mathrm{X}$-Rays of the cervical spine showed fusion of $\mathrm{C}_{2}-\mathrm{C}_{3}$. Magnetic resonance imaging showed absence of medullary stenosis; echocardiogram, abdominal ultrasound, electrocardiogram, and laboratorial exams without abnormalities. She was monitored with precordial stethoscope, electrocardiograph, pulse oximeter, non-invasive blood pressure, capnograph, and gas analyzer. Decreasing concentrations of sevoflurane $(8 \%$ to $4 \%$ ) and nitrous oxide at $50 \%$ were used for anesthetic induction. When the anesthetic plane was achieved, a venipunc- 
ture was performed with a $22 \mathrm{G}$ catheter. After administration of $7.5 \mathrm{mg}$ of atracurium and $35 \mu \mathrm{g}$ of fentanyl, conventional laryngoscopy was performed with the head of the patient in neutral position. With good visualization of the epiglottis and vocal cords (Cormack I), the patient was intubated without intercurrences. Anesthesia was maintained with $1 \%$ isoflurane and $50 \%$ nitrous oxide. During the procedure, $2.5 \mathrm{mg}$ of dexamethasone, $680 \mathrm{mg}$ of dipyrone, $2.5 \mathrm{mg}$ of ondansetron, and $35 \mu \mathrm{g}$ of fentanyl SC were administered. Despite the small technical difficulty, the surgery was performed without cervical extension. All monitoring parameters remained within normal limits during the surgery, which lasted approximately 20 minutes. At the end of the surgery, $680 \mathrm{mg}$ of neostigmine and 340 $\mathrm{mg}$ of atropine were administered and the patient was extubated without intercurrences. The patient remained stable in the post-anesthetic care unit, being discharged from the hospital on the first postoperative day.

\section{DISCUSSION}

In 1912, Maurice Klippel and André Feil reported the following anomalies found in a 46 years old patient: congenital fusion of cervical vertebrae leading to important limitation in the movement of the head and neck, short neck, and low hairline at the back of the neck. This condition became known as KFS ${ }^{2}$. Currently, we know that this is a complex syndrome that associates bone and visceral anomalies, and the triad described initially is present in less than $50 \%$ of the patients ${ }^{6}$. It has an incidence of 1:42,000 births and $60 \%$ are females. Its cause is unknown, being probably heterogeneous, with both genetic and environmental influences $^{2}$. Some investigators believe that its etiology is secondary to a defect on the PAX family gene. This would lead to failure in embryonic development between the third and the eighth weeks, resulting in lack of segmentation or faulty segmentation of mesodermal somites ${ }^{3}$. The genetic inheritance is transmitted in the majority of the cases sporadically, but autosomal recessive and rarely autosomal dominant traits have also been described ${ }^{8}$.

Varying degrees of vertebral involvement with different clinical presentations can be seen ${ }^{2}$. Several abnormalities, such as changes in atlantoaxial joint, stenosis of the medullary canal, and scoliosis can be associated. The cervical instability results in an increased risk of neurological spinal cord injury, even with minimal trauma. In a retrospective analysis of 21 patients with KFS, Nagib et al. observed that nine patients developed spontaneous, or with minimal trauma, neurologic deficits ${ }^{4}$.

Detailed clinical examination, including spinal X-rays, should be done before the surgery to know the extent of the spinal involvement. Diagnostic tests might be necessary to identify the presence of changes that might be associated with the syndrome, such as: cleft palate, ogival palate, facial asymmetry without dental changes, decrease in the inferior third of the face, deafness, both conductive and neural, and mental deficiency. Bone abnormalities that can generate ventilatory difficulties, such as shoulder changes, rib defects, and congenital elevation and shortening of the scapula (Sprengel deformity) are also common 2,7. Besides, changes in lung development, renal changes, and cardiac abnormalities can also be seen $2,7,8$. Cardiovascular abnormalities have an incidence of $4.2 \%$ to $14 \%$ in patients with KFS, and the defect in the interventricular septum is the most common. Patent ductus arteriosus, mitral valve prolapse, bicuspid aortic valve, and coarctation of the aorta leading to perioperative heart failure can also be present $4,7,9$. History of depression, drug abuse, and alcoholism are not uncommon in those patients ${ }^{8}$.

All changes seen in patients with KFS imply in judicious choice of the anesthetic technique. Restriction of the cervical movement and associated abnormalities might hinder the access to the airways, therefore complicating the anesthetic-surgical procedure. The most critical moments include laryngoscopy, intubation, and positioning of the patient for the surgery ${ }^{4}$. Although ventilation with a face mask is usually easy in those patients, the access to the airways represents a great challenge and requires previous planning. The anesthesiologist and surgeon should be extremely careful to avoid inappropriate cervical movements to prevent neurological damage. The successful use of tracheal intubation with fibrobronchoscopy ${ }^{1}$ and of the laryngeal mask ${ }^{3}$ have been reported. Fibrobronchoscopy is considered the safest technique. Awake intubation using fibrobronchoscopy have been used successfully by other authors ${ }^{5}$, but this technique requires patient cooperation, which is not always feasible, especially in children or patients with behavioral changes. Laryngeal mask is an easy option, and it has been used successfully in a patient with KFS who developed difficult to ventilate after anesthetic induction ${ }^{3}$. In the case presented here, although fibrobronchoscopy was available the authors decided to do a conventional laryngoscopy without cervical extension, and due to the perfect visualization of the vocal cords the patient was intubated. The laryngeal mask was not used to avoid hindering the surgical access since due to the position of the head in neutral position it was going to be very limited.

Farid et al. reported the case of a 45-year old female with KFS whose intubation was extremely difficult. In two prior occasions, she was intubated without difficulties: at 12 years of age for an appendectomy, and at 23 years of age for removal of an ovarian cyst. This report indicates that prior intubation does not imply in absence of difficulties in other procedures because cervical fusion is progressive ${ }^{8}$.

Down syndrome or Trisomy 21 is the most common chromosomal abnormality, and similar to KFS it also results in anatomical changes of the airways, such as: cleft lip and palate, narrow nasopharynx, and relatively large and protuberating tongue. The larynx and the cricoid ring tend to be small predisposing to acquired subglottic stenosis. Patients might have atlantoaxial subluxation, making neck extension risky ${ }^{1}$.

Tonsillectomy and adenoidectomy are the most common surgeries in ENT. The airways require special attention dur- 
ing those procedures, since it is involved in the surgical field. Bleeding, secretions, edema, and excessive manipulation might lead to respiratory complications. Frequent changes in the position of the head might be necessary, requiring perfect fixation of the tool used to access the airways ${ }^{10}$. Cervical complications related to incorrect positioning result in undesirable postoperative consequences, including cervical lesion in patients with risk factors ${ }^{11}$.

Although difficult airways are prominent on scientific information on KFS and DS, we did not find references to the association of both syndromes in the same patient. Contrary to what was expected, in the present case we did not find any difficulties to ventilate and intubate the patient. However, adequate equipment was available in case of difficulties in this process, and the expectation was to perform tracheal intubation with fibrobronchoscopy. Conventional laryngoscopy was performed previously to record the visualization according to the Cormack-Lehane scale with the head in neutral position. Considering the perfect visualization of the epiglottis and vocal cords, we decided to proceed with conventional intubation. However, the finding of easily accessible airways does not allow us to reduce the attention in those patients. The anesthesiologist should be able to recognize the difficulties and the risks involved in the approach of the airways in patients with congenital craniofacial disorders. Knowledge of the anatomical changes and adequate technical resources are paramount, especially in the case of oropharyngeal surgeries. The success of the procedure was based on the anticipation of possible problems and on the adequate interaction between the surgical and anesthetic teams. If the cervical malformation is the cause of cervical instability, with the consequent risk of nerve lesion, avoiding cervical mobilization, not only during airway management, but also during positioning of the patient and the surgery, can prevent this complication. 